\title{
Análise bibliométrica da produção científica brasileira sobre doença de Chagas
}

\section{Bibliometric analysis of the brazilian scientific production on Chagas disease}

\section{Análisis bibliométrico de la producción científica brasileña sobre la enfermedad de Chagas}

Hebert Luan Pereira Campos dos Santos ${ }^{1, a}$

ohebertluan@gmail.com | http://orcid.org/o000-0003-2722-7945

Taise de Alcantara Almeida ${ }^{2, b}$

taiseamancio@gmail.com | http://orcid.org/o000-0002-8917-9697

Hildebrando Antunes de Carvalho Neto, ${ }^{1, a}$

netoantuneso8@gmail.com | https://orcid.org/oooo-0002-0305-8473

Maria de Lourdes Lacerda Lemos, $, 2, b$

lourdes.mlll@hotmail.com | http://orcid.org/0000-0001-6093-687X

Josilene Silva Oliveira ${ }^{2, b}$

josinhaso@yahoo.com.br | http://orcid.org/0000-0002-7857-7665

Eliana Amorim de Souza ${ }^{1, c}$

amorim eliana@yahoo.com.br| http://orcid.org/0000-0002-9653-3164

Nília Maria de Brito Lima Prado, ${ }^{1,2, d}$

nilia.ufba@gmail.com | http://orcid.org/o00o-0001-8243-5662

${ }^{1}$ Universidade Federal da Bahia, Instituto Multidisciplinar em Saúde, Campus Anísio Teixeira. Vitória da Conquista, BA, Brasil.

2 Universidade Federal da Bahia, Instituto Multidisciplinar em Saúde, Programa de Pós-Graduação em Saúde Coletiva. Vitória da Conquista, BA, Brasil.

a Graduando em Medicina pela Universidade Federal da Bahia.

b Mestrado em Saúde Coletiva pela Universidade Federal da Bahia.

c Doutorado em Saúde Coletiva pela Universidade Federal do Ceará.

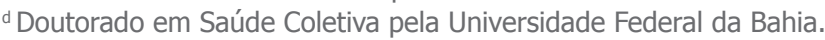

\section{RESUMO}

O presente estudo objetivou analisar a produção científica brasileira sobre a doença de Chagas a partir de publicações indexadas na base de dados Web of Science. Foram coletados e analisados os dados de 1.008 publicações no período entre 2006 e 2020. A interpretação dos dados permitiu identificar um expressivo crescimento da produção científica brasileira sobre a doença de Chagas e a abrangência da temática em periódicos internacionais. Contudo, há um enfoque nas áreas biomédicas do conhecimento com destaque para a Parasitologia e um diminuto número de investigações direcionadas às áreas da Saúde Pública, Ciências Sociais e Farmacêuticas. A bibliometria desvelou as lacunas ainda existentes na produção nacional e a necessidade de fortalecimento de políticas direcionadas a editais de pesquisa no país.

Palavras-chave: Doença de Chagas; Bibliometria; Produção científica; Brasil; Doenças negligenciadas. 


\section{ABSTRACT}

The present study aimed to analyze the Brazilian scientific production on Chagas disease from publications indexed in the Web of Science database. Data from 1,008 articles published between 2006 and 2020 were collected and analyzed. The interpretation of the data allowed the identification of an expressive growth of Brazilian scientific production on Chagas disease and comprehensiveness of the theme in international journals. However, there is a focus on the biomedical areas of knowledge with emphasis on Parasitology and a small number of investigations directed to the areas of Public Health, Social Sciences and Pharmaceuticals. The bibliometry revealed the gaps that still exist in the national production and the need to strengthen policies directed to research edicts in the country.

Keywords: Chagas disease; Bibliometrics; Scientific production; Brazil; Neglected diseases.

\section{RESUMEN}

El presente estudio tuvo como objetivo analizar la producción científica brasileña sobre la enfermedad de Chagas, a partir de las publicaciones indexadas en la base de datos Web of Science. Se recogieron y analizaron los datos de 1.008 artículos publicados entre 2006 y 2020. La interpretación de los datos permitió identificar un crecimiento expresivo de la producción científica brasileña sobre la enfermedad de Chagas y la amplitud del tema en las revistas internacionales. Sin embargo, hay un enfoque en las áreas biomédicas del conocimiento con énfasis en la Parasitología y un pequeño número de investigaciones dirigidas a las áreas de Salud Pública, Ciencias Sociales y Farmacéutica. La bibliometría reveló las lagunas que aún existen en la producción nacional y la necesidad de fortalecer las políticas dirigidas a los edictos de investigación en el país.

Palabras clave: Enfermedad de Chagas; Bibliometría; Producción científica; Brasil; Enfermedades desatendidas.

Este artigo compõe o dossiê Estudos métricos da informação científica em saúde, parte 1.

Contribuição dos autores:

Concepção e desenho do estudo: Hebert Luan Pereira Campos dos Santos, Taise de Alcantara Almeida, Hildebrando Antunes de Carvalho Neto, Maria de Lourdes Lacerda Lemos, Josilene Silva Oliveira, Eliana Amorim de Souza, Nília Maria de Brito Lima Prado. Aquisição, análise ou interpretação dos dados: Hebert Luan Pereira Campos dos Santos, Taise de Alcantara Almeida, Hildebrando Antunes de Carvalho Neto, Maria de Lourdes Lacerda Lemos, Josilene Silva Oliveira, Eliana Amorim de Souza, Nília Maria de Brito Lima Prado.

Redação do manuscrito: Hebert Luan Pereira Campos dos Santos, Taise de Alcantara Almeida, Hildebrando Antunes de Carvalho Neto, Maria de Lourdes Lacerda Lemos, Josilene Silva Oliveira, Eliana Amorim de Souza, Nília Maria de Brito Lima Prado. Revisão crítica do conteúdo intelectual: Hebert Luan Pereira Campos dos Santos, Taise de Alcantara Almeida, Hildebrando Antunes de Carvalho Neto, Maria de Lourdes Lacerda Lemos, Josilene Silva Oliveira, Eliana Amorim de Souza, Nília Maria de Brito Lima Prado.

Declaração de conflito de interesses: não há.

Fontes de financiamento: Fundação de Amparo à Pesquisa do Estado da Bahia (FAPESB).

Considerações éticas: não há.

Agradecimentos/Contribuições adicionais: FAPESB.

Histórico do artigo: submetido: 13 mar. 2021 | aceito: 25 ago. 2021 | publicado: 10 nov. 2021.

Apresentação anterior: não há.

Licença CC BY-NC atribuição não comercial. Com essa licença é permitido acessar, baixar (download), copiar, imprimir, compartilhar, reutilizar e distribuir os artigos, desde que para uso não comercial e com a citação da fonte, conferindo os devidos créditos de autoria e menção à Reciis. Nesses casos, nenhuma permissão é necessária por parte dos autores ou dos editores. 


\section{INTRODUÇÃO}

As doenças tropicais negligenciadas (DTN), apesar de impactarem fortemente as condições de saúde, receberam ao longo da história atenção insuficiente nas agendas governamentais de saúde e pesquisas internacionais e nacionais. Um importante marco foi a sua inclusão nas agendas dos Objetivos de Desenvolvimento do Milênio (ODM) e os Objetivos de Desenvolvimento Sustentável (ODS), que, alinhados à 'Agenda 2030 para o Desenvolvimento Sustentável', contemplaram as doenças negligenciadas (ONU, 2015). Contudo, o quadro epidemiológico e operacional de países da América Latina é desafiador pela complexidade do fenômeno que envolve dimensões econômicas, sociais, políticas, culturais, religiosas e jurídicas e devido à falta de definição de prioridades e estratégias de enfrentamento abrangentes (OLIVEIRA, 2018).

Dentre as DTN, a doença de Chagas é considerada uma das patologias de mais larga distribuição no continente americano, com elevada prevalência e expressiva morbimortalidade (SANGENIS et al., 2016). Os dados apontam cerca de 7 milhões de infectados na América Latina, com uma média anual de 30 mil casos novos e de 14 mil óbitos pela doença. Estima-se ainda que 70 milhões de pessoas permanecem expostas ao risco de contrair a doença em 21 países endêmicos no território das Américas (BRASIL, 2020a). Essa patologia persiste como um problema concreto e impactante na América Latina sob múltiplos aspectos vinculados, entre outros, às iniquidades sociais. Além disso, com o advento da globalização, outros países não endêmicos tornam-se passíveis à doença (DIAS, 2007).

No Brasil, entre os anos de 2015 e 2019 foram registrados 22.154 óbitos, cuja causa básica foi a doença de Chagas (DATASUS, c2020). Tais resultados demonstram a magnitude da doença no país. Diante desse cenário, o Brasil propôs o Protocolo Clínico de Diretrizes Terapêuticas (PCDT), cujo intuito é ampliar o rastreamento e tratamento de pessoas acometidas pela doença nas fases aguda e crônica (BRASIL, 2018a), e a inclusão da doença de Chagas crônica no rol de doenças de notificação compulsória no país (BRASIL, 2020b).

Ante a esse cenário, evidencia-se que ainda é incipiente a sinergia entre pesquisa, desenvolvimento e inovação e as ações estratégicas de saúde em torno das DTN. Dessa forma, reconhece-se a necessidade de avanços no que concerne ao planejamento operacional direcionado à ampliação de articulações com universidades e centros de pesquisas, com o intuito de viabilizar por meio de pesquisas aplicadas a inclusão das estratégias pelos serviços, o controle sistemático e a diminuição da morbimortalidade causada pela doença, especialmente, nos países mais atingidos (SOBRAL; SILVA; MIRANDA, 2017).

Ademais, há uma lacuna na literatura quanto à análise bibliométrica sobre a doença de Chagas (HAYASHI et al., 2007), apesar de constituir uma metodologia fundamental para a avaliação da produtividade e a qualidade de referentes da produção científica desta temática no Brasil (VIEIRA; SANNA, 2013; FILIPPO; FERNÁNDEZ, 2002). Considerando o exposto, este artigo objetiva identificar e caracterizar a produção científica brasileira sobre a doença de Chagas, utilizando dados bibliográficos indexados na base de dados da Web of Science (WoS).

\section{METODOLOGIA}

Realizou-se uma análise bibliométrica da produção científica no Brasil sobre a doença de Chagas, buscando verificar e analisar especificidades acerca das tendências de crescimento, dispersão e obsolescência do conhecimento sobre as publicações na temática.

O percurso metodológico para o planejamento, coleta e análise dos dados envolveu as seguintes etapas: a) definição da fonte de informação; b) levantamento bibliométrico; c) descrição dos dados obtidos; e d) análise dos resultados. 
A fonte de informação selecionada para o estudo foi a base de dados científicos Web of Science (WoS), devido à natureza multidisciplinar e por englobar um conjunto de dados de periódicos científicos de alto impacto, permitindo uma análise avançada, referente ao número de publicações, ano, autores, idiomas, entre outros.

Como estratégia debuscautilizou-se a associação dos termos: 'Chagas Disease', considerando ser terminologia de busca padrão, e 'Brazil'. A delimitação temporal compreendeu o período de 1 de janeiro de 2006 a 28 de dezembro de 2020. Partiu-se de 2006 por ser esse o ano em que o Brasil alcançou certificação de eliminação do mais importante vetor na cadeia de transmissão em seu território, o Triatoma infestans, pela Organização Pan-Americana de Saúde (OPAS) (VILLELA et al., 2009), chegando, então, até o ano 2020 (os últimos quinze anos de produção científica), sob a tentativa de identificar possíveis lacunas que possam subsidiar a adoção de medidas para o controle e cuidado à saúde referentes à infecção pelo Trypanossoma cruzi.

Os critérios de inclusão foram publicações disponíveis na íntegra, cujo pelo menos um dos autores estivesse filiado às instituições de ensino ou pesquisa brasileiras. Não houve exclusão a priori de nenhuma abordagem metodológica, tendo sido incluídos tanto textos qualitativos quanto quantitativos. Foram excluídas publicações que não estavam disponíveis on-line na base de dados priorizada ou que foram publicados em período anterior ao pré-definido e cujos autores estivessem vinculados somente a instituições de ensino e pesquisa de outros países.

Identificaram-se 1.008 publicações, das quais foram sistematizados os principais dados quantitativos identificados nas ferramentas da WoS, a Science Citation Index - Expanded (SCI); Social Science Citation (SSCI) e Conference Science Citation Index (CSCI), por tipologias de indexação, quais sejam: tipo de documento, distribuição por ano, principais periódicos, idioma de publicação, cooperação internacional, autores, áreas do conhecimento, instituições e agências de financiamento. A análise desses dados considerou até a décima colocação de cada categoria com ressalvas à variável correspondente aos autores, para a qual foram utilizados os 25 principais classificados no ranking. Ademais, para a investigação da área de estudo desses pesquisadores, utilizou-se a Plataforma Lattes (CNPQ, c2020), já os currículos de indivíduos estrangeiros não disponíveis nesta plataforma foram acessados em um site institucional, o Albert Einstein College of Medicine (EINSTEIN, c2020).

Extraídas as informações bibliométricas para a análise dos dados, procedeu-se a compilação quantitativa com organização dos dados em planilhas do Microsoft Excel, analisados por intermédio de medidas de frequência absoluta e/ou médias simples e/ou percentuais representados por gráficos e quadro contendo as sínteses.

\section{RESULTADOS E DISCUSSÃO}

Das 1.008 publicações analisadas, 90,38\% eram artigos originais, e os demais corresponderam a estudos de revisão, documentos técnicos e capítulo de livros, conforme distribuição da frequência de publicações por ano (Figura 1). 


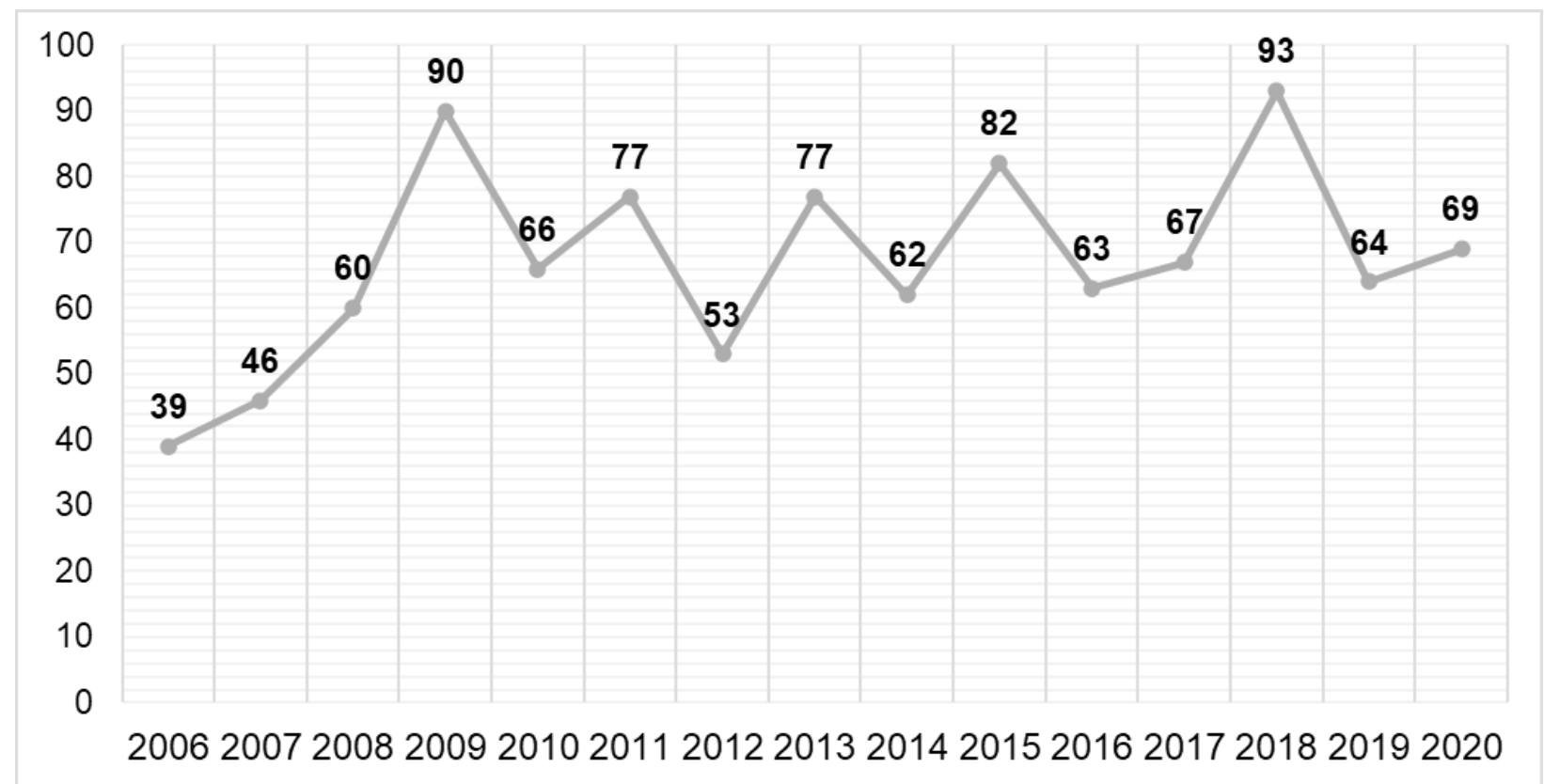

Figura 1 - Frequência absoluta das publicações sobre doença de Chagas por ano indexadas na base de dados WoS, 2006 a 2020

Fonte: elaboração dos autores.

Sobre o total das publicações, os dados temporais revelaram uma média simples da produção anual correspondente a 67,2 manuscritos/ano, com as menores frequências nos dois primeiros anos da análise, 2006 e 2007, respectivamente 39 e 46 publicações que galgaram aumentos relevantes a posteriori. Cabe ressaltar que em 2006 o Brasil foi certificado pela Organização Pan-Americana de Saúde pela interrupção da cadeia de transmissão pelo vetor da espécie Triatoma infestans (VILLELA et al., 2009). As estratégias de controle empreendidas no contexto brasileiro em articulação com as mudanças socioeconômicas foram responsáveis pela transformação do perfil epidemiológico da infecção pelo Trypanossoma cruzi (DIAS et al., 2016a). Tal fato pode ter possibilitado uma ampliação da atenção à saúde e das pesquisas científicas sobre a temática.

De maneira geral, um fator que colaborou para o aumento do número de publicações científicas a partir de 2007 no Brasil foi a criação do Programa de Apoio a Planos de Reestruturação e Expansão das Universidades Federais, cuja finalidade era ampliar o acesso e evitar a evasão do ensino superior interferindo positivamente na capacidade de publicação das Instituições Públicas de Ensino Superior (BRASIL, 2007).

Os dados apontaram, ainda, que o segundo maior período de elevação do número de produções ocorreu em 2009, correlacionado à comemoração do centenário da descoberta da doença por Carlos Chagas em 1909. Esse contexto contribuiu para o retorno da temática aos debates midiáticos e pode ter fomentado o interesse e a intensificação de investigações e publicações científicas concernentes à doença de Chagas, reforçando a necessidade de ampliar investigações direcionadas às populações acometidas nos territórios de maior endemicidade (ANDREOLLO; MALAFAIA, 2009; CALDAS, 2008).

No ano de 2015, a Organização das Nações Unidas pactuou com os países membros os objetivos que compõem a agenda 2030, dentre eles, destaca-se o objetivo de reduzir as enfermidades transmissíveis e eliminar as DTN (ONU, 2015). Em consonância direta com a agenda 2030, a OPAS, do mesmo modo, estabeleceu compromisso semelhante na agenda de saúde sustentável para as Américas no período compreendido entre 2018 e 2030 (OPAS, 2017). Infere-se que os esforços globais e regionais dirimidos para o enfrentamento das DTN tenham despertado a manutenção/aumento de investigações acerca dessas 
doenças, a exemplo da doença de Chagas. Neste contexto, nota-se que o ano de 2018 foi responsável pela maior quantidade de publicações sobre este assunto.

Assim, a análise dos dados permitiu constatar, por meio do número de publicações por ano, a evolução da pesquisa sobre doença de Chagas. Oliveira e Leite (2016) destacaram que embora as publicações sobre o tema tenham iniciado em 1948, a maior parte foi produzida a partir dos anos 2000, o que indica uma produção relativamente recente sobre a temática.

Em relação à representatividade da temática, um estudo que buscou avaliar a produção científica latinoamericana sobre Medicina Tropical na WoS identificou que a doença de Chagas assumiu o segundo lugar dentre as publicações em DTN, superada apenas por produções relacionadas à leishmaniose (SOBRAL; SILVA; MIRANDA, 2017). Em outra investigação, que contemplou a análise das produções sobre DTNs em revistas cientificas, demonstrou-se que dos 669 periódicos abarcados, a leishmaniose está presente em 282, e a doença de Chagas em 237, reforçando o lugar de destaque dessas doenças na produção de conhecimento (SOBRAL et al., 2020).

Quanto à distribuição de publicações sobre o tema por país, Sobral, Silva e Miranda (2017) constataram que o Brasil foi responsável pela maioria (59\%) dos manuscritos entre as nações da América Latina. Ressaltase ainda que o Brasil é o terceiro país com maior produção científica na área, número que surpreende, considerando que a coleção da WoS indexa em maior amplitude publicações internacionais de países desenvolvidos (SOBRAL et al., 2016). Com isto, ressalta-se a importância da liderança brasileira, haja vista a produção científica em DTN não ser prioritária para os países desenvolvidos, como EUA e países da Europa, dada a endemicidade da doença ocorrer em países em desenvolvimento, a maioria localizada na região latino-americana (SOBRAL; SILVA; MIRANDA, 2017).

No que se refere às revistas cientificas que mais publicaram sobre a doença de Chagas na WoS (Figura 2), dois periódicos brasileiros destacaram-se em número de publicações, a Revista da Sociedade Brasileira de Medicina Tropical e a revista Memórias do Instituto Oswaldo Cruz, dado também identificado por Sobral e colaboradores (2020).

Dos 10 periódicos com maior número de publicações, seis são internacionais. Assim, percebe-se que, apesar de o Brasil ser um dos maiores produtores de conhecimento sobre a doença de Chagas, a presença de periódicos nacionais é inferior à dos internacionais (OLIVEIRA; LEITE, 2016). 


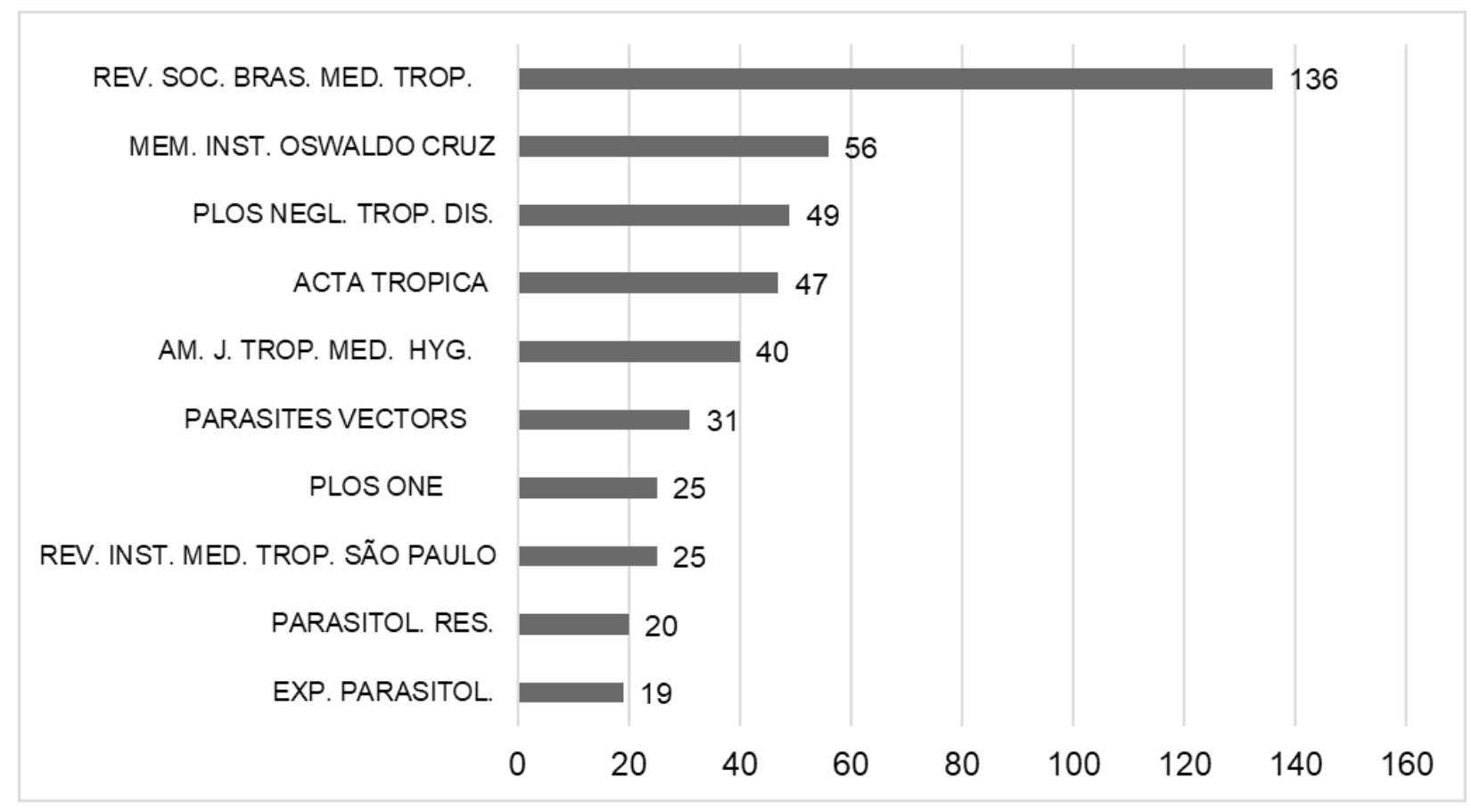

Figura 2 - Frequência absoluta das publicações sobre doença de Chagas por periódicos na base de dados WoS, 2006 a 2020

Fonte: elaboração dos autores.

O sistema de avaliação brasileiro sobre a produção científica derivada dos Programas de Pós-graduação não favorece as publicações em revistas nacionais, o que contribui para a incipiência de periódicos brasileiros com estratos Qualis mais elevados, direcionando a preferência dos autores para publicações em periódicos internacionais de maior impacto científico. Com isto, os pesquisadores condicionam a sua produção a periódicos com maior privilégio no contexto internacional, o que gera menor reconhecimento interno acerca dos problemas de regiões endêmicas (SOBRAL et al., 2020). Percebe-se, também, que há uma tentativa das revistas nacionais em seguir os padrões internacionais buscando visibilidade, reconhecimento e autoafirmação no cenário da comunicação científica dos países desenvolvidos.

Dias (2007) sinaliza a preocupação com o desestímulo em relação às investigações sobre o controle da transmissão e o manejo de infectados pelo Trypanossoma cruzi face à dificuldade de publicação em revistas com alta qualidade acadêmica nacionais. No entanto, reforça-se que as produções que tratam sobre as doenças negligenciadas lidam com um recorte geopolítico bem definido e, na maior parte, circunscritas ao conjunto de países em desenvolvimento.

Quando analisado o idioma original das publicações, notou-se que 90,08\% dos artigos, foram publicados em inglês (908); 8,43\% em português, correspondendo a 85 publicações; 1,19\% em espanhol (12); e apenas $0,3 \%$ em francês (3). Observa-se uma tendência mundial em estimular os autores a publicarem sobre temáticas de abrangência mundial e em periódicos indexados em bases de dados internacionais em língua inglesa ante a possibilidade de visibilidade em escala global (LEE; LEE, 2013). Meneghini e Packer (2007) constataram que os pesquisadores do Brasil publicam em torno de 50.000 artigos/ano, dos quais cerca de 60\% são em português. Desse percentual, aproximadamente 18.000 artigos são publicados em revistas indexadas na WoS. No entanto, somente 2,7\% desses manuscritos estão na língua portuguesa (MENEGHINI; PACKER, 2007).

Um estudo argentino comparou o efeito da linguagem no número de citações de artigos publicados em seis periódicos de ciências naturais de cinco países que publicaram artigos em inglês ou em outros idiomas. Evidenciou-se que os artigos veiculados na língua inglesa têm maior número de citações do que os 
publicados em outros idiomas, sugerindo maior probabilidade de ser citado globalmente pela comunidade científica (BITETTI; FERRERAS, 2017).

Ao verificar os países que cooperam com as pesquisas desenvolvidas por autores e instituições brasileiras, observa-se que os Estados Unidos possuem 15,18\% de coautoria (153), a Argentina aparece com 5,45\% (55), a França com 3,97\% (39), Colômbia apresenta 3,77\% (38), Inglaterra e Espanha, respectivamente, 2,78\% (28) e 2,48\% (25), e os demais países apresentaram percentuais menores que $2 \%$.

Em estudo que analisou a produção científica sobre doença de Chagas a partir da base de dados da PubMed (1940-2009), González-Alcaide et al. (2012) constataram que o Brasil é a principal referência da América do Sul no assunto, sendo o país com o maior número de pesquisadores envolvidos nas principais redes de colaboração científica sobre a temática no mundo. Sobral et al. (2016) e Sobral, Silva e Miranda (2017) ao traçarem o perfil da produção científica na América Latina sobre Medicina Tropical, área da medicina que lida com problemas de saúde de mais difícil controle nas regiões tropicais ou subtropicais, perceberam que as relações de cooperação científica são mais intensas entre o Brasil e os Estados Unidos.

As pesquisas realizadas pelo Relatório Research in Brazil demonstram que os documentos resultantes de coautorias internacionais têm tido um maior impacto do que a pesquisa realizada exclusivamente no Brasil, o que reafirma a importância da cooperação entre as nações na construção e divulgação do conhecimento (CROSS; THOMSON; SIBCLAIR, 2017).

O quadro 1 apresenta a frequência absoluta de publicação pelos 25 autores que emergiram na busca da WoS e suas respectivas áreas de estudo.

\section{Quadro 1 - Frequência absoluta entre as publicações sobre doença por autores na base de dados WoS, 2006 a 2020, e suas respectivas áreas de estudo}

(continua)

\begin{tabular}{|c|c|c|}
\hline Autores & $\begin{array}{l}\text { Número de } \\
\text { publicações }\end{array}$ & Área de estudo \\
\hline Ana Maria Jansen & 49 & Parasitologia/Protozoologia \\
\hline Liléia Diotaiuti & 43 & Parasitologia/Entomologia \\
\hline João Aristeu da Rosa & 39 & Parasitologia/Entomologia \\
\hline Jane Costa & 31 & Zoologia/Entomologia \\
\hline Marli Maria Lima & 30 & Parasitologia \\
\hline Carlos Eduardo Almeida & 28 & Entomologia \\
\hline João Carlos Pinto Dias & 27 & Medicina Tropical/Infectologia \\
\hline Alberto Novaes Ramos Júnior & 26 & Saúde Coletiva/ Medicina Tropical/Infectologia \\
\hline Antônio Luiz Pinho Ribeiro & 26 & Cardiologia/Infectologia/Medicina Tropical \\
\hline Hebert Bernard Tanowitz* & 26 & Parasitologia/Entomologia \\
\hline Rodrigo Gurgel-Gonçalves & 25 & Protozoologia/Entomologia \\
\hline Ester Cerdeira Sabino & 21 & Imunologia \\
\hline Teresa Cristina M. Gonçalves & 20 & Parasitologia/Entomologia \\
\hline Egler Chiari & 19 & Parasitologia/Protozoologia \\
\hline Louis M. Weiss & 19 & Patologia/Infectologia \\
\hline Otília Sarquis & 18 & Parasitologia/Entomologia \\
\hline Vagner José Mendonça & 17 & Parasitologia/Entomologia \\
\hline Kaio Cesar Chaboli Alevi & 16 & Entomologia/Genética \\
\hline José Rodrigues Coura & 16 & Medicina Tropical \\
\hline Jorg Heukelbach & 16 & Farmacologia/Medicina Tropical \\
\hline Linda A. Jelicks & 16 & Fisiologia \\
\hline Francisco Rogerlândio Martins-Melo & 16 & $\begin{array}{l}\text { Saúde Coletiva/Epidemiologia/ } \\
\text { Doenças Negligenciadas }\end{array}$ \\
\hline
\end{tabular}




\begin{tabular}{|l|l|l|}
\hline Autores & $\begin{array}{l}\text { Número de } \\
\text { publicações }\end{array}$ & Área de estudo \\
\hline Mônica Lúcia Gomes & 15 & Parasitologia/Biologia Molecular \\
\hline Aluízio Prata & 15 & Medicina tropical/Infectologia \\
\hline $\begin{array}{l}\text { André Luiz Rodrigues Roque } \\
\text { *In memoriam }\end{array}$ & 15 & Parasitologia \\
\hline
\end{tabular}

Fonte: elaboração dos autores.

No ranking dos 25 principais pesquisadores com publicação sobre doença de Chagas no Brasil (2006 a 2020), apenas nove (36\%) são mulheres. De acordo com Carvalho, Coeli e Lima (2018), não há uniformidade no número de mulheres no mundo da ciência, visto que o grupo constitui apenas $15 \%$ dos pesquisadores. Por sua vez, o estudo ‘Gender in the Global Research Landscape’ publicado em 2017 pela Elsevier realizou uma análise do desempenho da pesquisa e identificou que, dentre os países pesquisados, Brasil e Portugal são os que mais contam com pesquisadoras do sexo feminino em trabalhos científicos ( $49 \%$ do total), quase a mesma quantidade que os pesquisadores homens (ELSEVIER, 2017). Corroborando com esta constatação, o Gráfico 3 apresenta a distribuição do número de publicações pelas principais áreas de pesquisa indexadas na base de dados WoS.

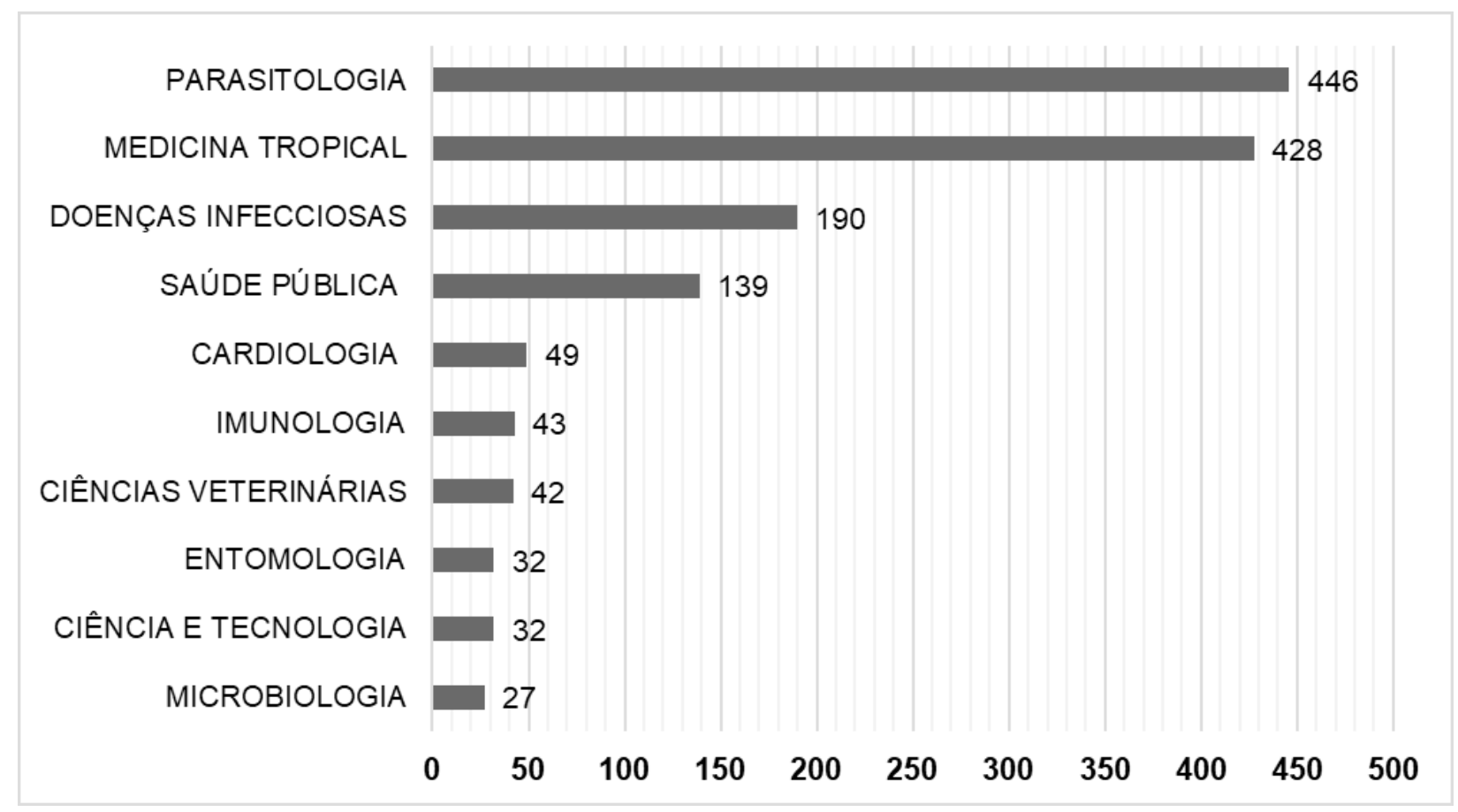

Figura 3 - Frequência absoluta das publicações científicas sobre doença de Chagas por área de pesquisa indexadas na base de dados WoS, 2006 a 2020

Fonte: elaboração dos autores.

Evidencia-se que a área referente a Parasitologia apresentou maior quantidade de publicações, um total de 446. Tal superioridade relaciona-se ao fato de a área em questão abranger o estudo de parasitas como o Trypanossoma cruzi, causador da doença de Chagas; em seguida, aparece a Medicina Tropical com 428 publicações, o que pode ser justificado por se tratar de uma doença característica dos países tropicais e subtropicais. Esses dados aproximam-se dos resultados encontrados por Silva e Luz Junior (2016), que verificaram correlação da área de Parasitologia com a alta produtividade nesta temática. Outro estudo 
apontou a consolidação da área da Protozoologia como área que mais retém as pesquisas sobre o assunto (KROPF; AZEVEDO; FERREIRA, 2000).

Sob outra perspectiva, observa-se que a área da Saúde Pública, necessária para fomentar as políticas e ações direcionadas ao controle da doença de Chagas, foi contemplada em apenas 139 publicações, um número muito reduzido quando comparado às duas áreas supracitadas.

Um estudo bibliométrico realizado por Pellegrini, Goldbaum e Silvi (1997) verificou que a produção epidemiológica brasileira se concentra em torno dos temas relacionados às doenças infecciosas e saúde maternoinfantil, enquanto na produção mundial, as doenças crônicas apresentam um maior destaque (PELLEGRINI; GOLDBAUM; SILVI, 1997). Tais dados reforçam o comprometimento dos pesquisadores brasileiros na busca pela ampliação dos conhecimentos sobre os problemas de saúde pública que persistem no país.

Desde os primeiros estudos em 1909, grande parte das pesquisas realizadas sobre a infecção por Trypanossoma cruzi, tanto em cenário brasileiro, quanto internacional, envidou esforços referentes ao estágio agudo da doença. Entretanto, os desafios para confirmação do estágio crônico da infecção demonstraram que o desenvolvimento de estudos socioepidemiológicos é relevante para a identificação de novas alternativas diagnósticas, uma vez que essa fase da doença pode ocasionar consequências sociais relacionadas a óbitos súbitos e incapacidade total ou parcial para as atividades laborais, principalmente, relacionadas às implicações cardíacas (KROPF; AZEVEDO; FERREIRA, 2000). Destarte, mais de 80\% das pessoas atingidas pela doença no mundo não têm acesso ao diagnóstico e tratamento (DIAS et al., 2016b), dado que reforça a necessidade de reflexões críticas sobre a temática, em especial, pela área de pesquisa em saúde pública.

Ainda cabe mencionar que a grande área das Ciências Humanas e Sociais (Sociologia, Antropologia, entre outras) representou uma diminuta parcela nas publicações científicas da WoS sobre a doença de Chagas. Nesse sentido, o desenvolvimento de estudos nessas áreas apresenta relevância ante a necessidade de estabelecer uma compreensão aprofundada da importância social da doença (MIOTTO; BARCELLOS, 2009).

Similarmente, a Farmacologia não aparece entre as principais áreas de conhecimento nas produções analisadas. Mesmo com grandes avanços no controle; no conhecimento adquirido ao longo do tempo sobre o ciclo de vida, metabolismo e genética do Trypanossoma cruzi; e nos adventos da tecnologia farmacêutica, não se vislumbra a erradicação da doença por meio de investimentos robustos em terapias medicamentosas (DIAS et al., 2016a; MALAFAIA, 2009). Apesar do predomínio de áreas biomédicas nas publicações sobre a infecção, o tratamento medicamentoso disponível para a população infectada ainda apresenta reduzida eficácia e alta toxicidade na fase crônica, fato que pode justificar a necessidade de ampliação do desenvolvimento de estudos na área das Ciências Farmacêuticas e Biotecnológicas (WESTPHALEN et al., 2012). Aspectos relacionados ao acesso limitado à confirmação diagnóstica e à terapia precoce voltado às DTN demonstram a necessidade de soluções que venham a suprir essas lacunas, não somente no espaço científico, mas no mercado, e especialmente, na saúde pública (BRASIL, 2018b).

A Figura 4 apresenta as relações entre os manuscritos publicados na WoS no período delimitado e as principais instituições de vinculação dos autores. 


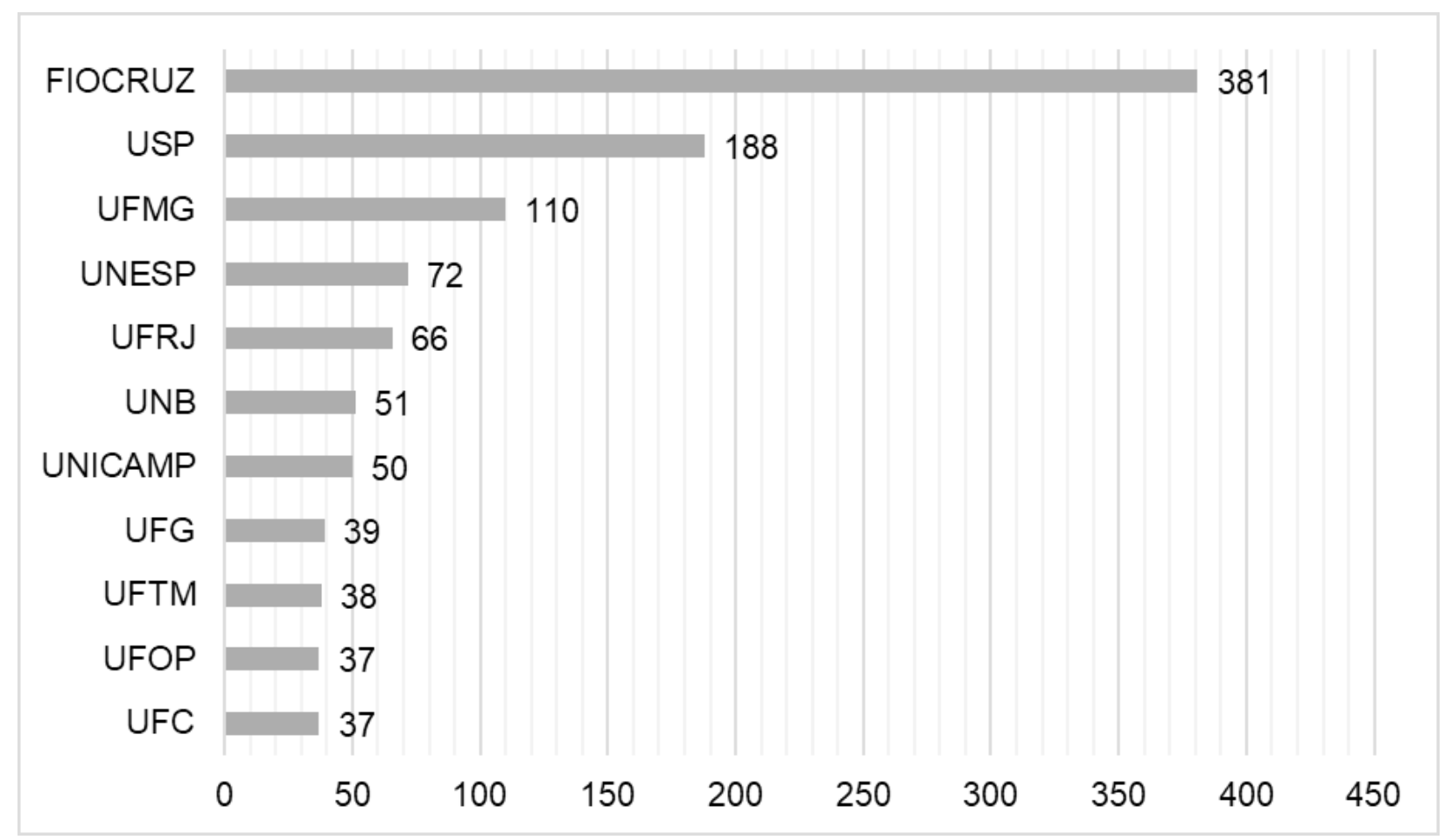

Figura 4 - Frequência absoluta das publicações sobre doença de Chagas por instituições na base de dados WoS, de 2006 a 2020

Fonte: elaboração dos autores.

Ao observar as afiliações dos autores das publicações é possível verificar que, entre as 10 instituições, a Fundação Oswaldo Cruz (Fiocruz) assume o primeiro lugar do ranking em consonância com pesquisa de Sobral, Silva e Miranda (2017), que avaliou as publicações sobre Medicina Tropical na WoS e identificou intensa relação entre a temática envolvendo a doença de Chagas e as produções derivadas da Fiocruz (RJ).

No que se refere ao aspecto regional, nenhuma instituição pertence diretamente às regiões Norte e Sul do Brasil, e apenas uma é vinculada ao Nordeste - Universidade Federal do Ceará, na $10^{\text {a }}$ posição com 37 publicações, apresentando performance semelhante à Universidade Federal de Ouro Preto. A região Sudeste abarcou o maior número (7 das 11 instituições elencadas), seguida pela região Centro-Oeste com 2 instituições. Os três estados localizados na região Sudeste (RJ, SP e MG) apresentaram maior número de publicações. Este dado corrobora com o Relatório de Pesquisa no Brasil, que evidenciou concentração da produção nos três estados citados, todavia, majoritariamente em São Paulo, seguido de Rio de Janeiro e Minas Gerais (CROSS; THOMSON; SIBCLAIR, 2017).

Vale ressaltar que a Fiocruz é uma instituição presente em todas as regiões do país, e suas publicações podem contemplar diversos estados, havendo a necessidade de estudos futuros desmembrarem as produções para uma análise de desempenho loco-regional.

Paradoxalmente, a distribuição regional dos casos confirmados da doença, entre os anos de 2012 e 2016, evidenciou um total de 1190 casos confirmados no Brasil, exibindo maior número no Norte do país com 1156 confirmações, seguidos de 24 no Nordeste, 4 no Sudeste, 3 no Sul e 3 no Centro-Oeste (BRASIL, 2019). Os dados descritos demonstraram uma lógica inversa entre as regiões com maior produção cientifica sobre o tema e de maior número de casos da infecção pelo Trypanossoma cruzi, o que denota que o território latino-americano tem sido atingido pelas doenças tropicais, mas não é evidenciada uma proporcionalidade do cenário epidemiológico favorável à produção de conhecimento nos países, o que pode ser direcionado às regiões do Estado brasileiro (SOBRAL; SILVA; MIRANDA, 2017). 
No que se refere aos indicadores coletados pelo Geocapes, aplicativo gráfico que exibe informações quantitativas dos dados relativos à pós-graduação stricto sensu com precisão geográfica, nota-se que os estados de São Paulo (929), Rio de Janeiro (503) e Minas Gerais (469) possuem o maior número de programas de pós-graduação do país, e lideram outros indicadores, como investimentos da Coordenação de Aperfeiçoamento de Pessoal de Nível Superior (Capes) em editais de fomento e bolsas científicas, inclusive, de pós-graduação (CAPES, c2020). Tais dados podem explicar a relação assimétrica das publicações ao considerar-se a origem, instituições de ensino superior ou centros de pesquisa, dado aos distintos montantes de recursos financeiros para o fomento de pesquisas.

Ao analisar a frequência absoluta das publicações associadas às agências de financiamento, o Conselho Nacional de Desenvolvimento Científico e Tecnológico (CNPq) e a Capes perfazem 470 (31,1\%). Pode-se destacar também as Fundações de Amparo à Pesquisa dos Estados de São Paulo, Rio de Janeiro e Minas Gerais entre as dez principais agências financiadoras vinculadas a estados brasileiros com instituições de ensino superior e de pesquisa que mais publicam sobre a temática. Evidencia-se no ranking apresentado, no recorte temporal delimitado ao estudo, uma forte participação de agências internacionais no financiamento de atividades de pesquisa direcionadas à doença de Chagas.

De um modo geral, por investigação induzida e por demanda espontânea, as últimas quatro décadas desvelam um crescimento exponencial na pesquisa em doença de Chagas, o que resultou em notório desenvolvimento científico dos países afetados. Observa-se que no período analisado por este artigo, apesar de alguns órgãos de fomento à pesquisa governamental destinarem editais de apoio às DTN (DIAS et al., 2016a), foi notória a baixa priorização de financiamentos direcionados a pesquisas relacionadas com a doença de Chagas humana, principalmente, àquelas de natureza aplicada, focadas nos aspectos organizacionais, de controle e manejo da doença. Ademais, os incentivos existentes derivam, basicamente, de um grande esforço da comunidade científica latino-americana (DIAS, 2007; SILVEIRA et al., 2002).

O cenário político atual do Brasil de austeridade fiscal desencadeou a redução dos recursos financeiros destinados ao apoio e ao desenvolvimento de pesquisas científicas no Brasil em diversas áreas, inclusive, referentes às doenças tropicais negligenciadas. Esse contexto sinaliza a recrudescência de iniquidades em saúde, principalmente, pela anomia da população exposta ou afetada pelo mal, que vive em bolsões de pobreza onde subsistem riscos de transmissão e se concentram indivíduos infectados, sem adequada atenção à saúde (DIAS, 2007).

\section{CONCLUSÃO}

A análise da produção científica sobre Doença de Chagas na WoS permitiu constatar um crescimento da produção sobre o tema com expansão considerável a partir do ano de 2009, dada a complexidade das modificações na caracterização epidemiológica e da morbimortalidade relacionada à doença.

Ao observar os dados coletados, cabe destacar que, apesar do desenvolvimento científico sobre a doença de Chagas no país, muitas lacunas ainda necessitam ser preenchidas, principalmente, no que se refere à ampliação do fomento financeiro para realização de pesquisas sobre as DTN; à necessidade de orientação das pesquisas em DTN aos aspectos locais, privilegiando os países endêmicos; ao incentivo à realização de pesquisas relacionadas a novas tecnologias e terapias medicamentosas; e à elaboração de medidas políticas sistemáticas que direcionem práticas de saúde pública para o controle da doença.

Reconhecer a verdadeira dimensão da negligência deve pressupor questionar criticamente racionalidades que informam modos de operar políticas, que, a despeito de indiscutíveis avanços em termos de saúde pública, mantêm regras e contornos nos marcos da subalternidade e da dependência de agendas globais. É importante destacar que o investimento em pesquisa é fundamental para o país e constitui a alavanca para geração de conhecimentos qualificados para subsidiar a implementação de ações consistentes e 
sustentáveis de gestão, vigilância, controle e atenção à saúde e ao social, que atendam aos princípios de coerência, eficácia e efetividade (DIAS et al., 2016a).

Ademais, percebe-se a necessidade de ampliar a realização de pesquisas sobre doença de Chagas no âmbito das áreas de Saúde Pública, Ciências Sociais e Farmacêuticas, dada a complexidade biopsicossocial da doença, inclusive, o estigma em torno dos usuários, em razão de maior correlação com iniquidades sociais, assim como a necessidade de avançar no que concerne ao tratamento etiológico. Cabe destacar a importância e necessidade de colaboração entre essas e outras áreas do conhecimento que, de forma interdisciplinar com o campo da saúde, possam reforçar reflexões acerca da prevenção e combate à doença.

Uma lacuna desse estudo encontra-se nos aspectos metodológicos referentes à restrição da seleção de publicações apenas a uma base de dados científica, e a priorização de estratégias de busca utilizadas, que compreendeu apenas dois descritores em saúde, bem como, a inclusão apenas de publicações disponíveis na íntegra. Em consequência desse conjunto de opções metodológicas, alguns artigos relevantes, que porventura pudessem ter sido abarcados com o uso de outros descritores correspondentes (por exemplo, Trypanossoma Cruzi) e outros tipos de publicações, como teses e dissertações, anais de congressos, podem não ter sido contemplados pela amostra analisada. Apesar das limitações, os dados selecionados apontaram resultados importantes acerca do crescimento na produção científica sobre a temática, indicando uma maior preocupação e atenção dos pesquisadores com a geração de conhecimento científico sobre o tema, com o objetivo de otimizar as estratégias de enfrentamento da doença.

Por fim, os resultados são pertinentes ao sinalizarem a necessidade de desenvolvimento de ações coletivas lideradas por países com maior incidência da doença, ampliando a entrada na agenda governamental específica que contemple particularidades e questões derivadas de estudos loco-regionais, possibilitando fomentar a formulação de políticas de saúde e a implementação das ações e serviços de saúde pública direcionados a um controle efetivo das DTN, especialmente a doença de Chagas.

\section{REFERÊNCIAS}

ALBERT EINSTEIN COLLEGE OF MEDICINE (EINSTEIN). Departments \& Centers - Faculty Directory. New York: Einstein, c2020. Disponível em: https://einsteinmed.org/education/. Acesso em: 10 dez. 2020.

ANDREOLLO, Nelson Adami; MALAFAIA, Osvaldo. Os 100 anos da doença de Chagas no Brasil. ABCD. Arquivos Brasileiros de Cirurgia Digestiva (São Paulo), São Paulo, v. 22, n. 4, p. 185-191, 2009. DOI: https://doi.org/10.1590/S0102-67202009000400001. Disponível em: https://www.scielo.br/j/abcd/a/pM7GyKjbn vRLGNCzFCcMwVz/?lang=pt. Acesso em: 22 set. 2021.

BITETTI, Mario S. Di.; FERRERAS, Julián A. Publish (in English) or perish: the effect on citation rate of using languages other than English in scientific publications. Ambio, Estocolmo, v. 46, n.1, p. 121-127, 2017. DOI: https://doi.org/10.1007/s13280-016-0820-7. Disponível em: https://link.springer.com/article/10.1007\%2 Fs13280-016-0820-7. Acesso em: 15 mar. 2019.

BRASIL. Decreto n 6096, de 24 de abril de 2007. Institui o Programa de Apoio a Planos de Reestruturação e Expansão das Universidades Federais - REUNI. Diário Oficial da União, Brasília, DF, p. 7, 25 abr. 2007. Disponível em: http://www.planalto.gov.br/ccivil_03/_Ato2007-2010/2007/Decreto/D6096.htm. Acesso em: 5 jan. 2021.

BRASIL, Ministério da Saúde. Secretaria de Ciência, Tecnologia e Insumos Estratégicos. Protocolo Clínico e Diretrizes Terapêuticas Doença de Chagas Brasil. Brasília, DF: O Ministério, out. 2018a. Disponível: http:// conitec.gov.br/images/Protocolos/Relatorio PCDT Doenca de Chagas.pdf. Acesso em: 10 abr. 2019.

BRASIL. Ministério da Saúde. Secretaria de Vigilância em Saúde. Saúde Brasil 2017: uma análise da situação de saúde e os desafios para o alcance dos objetivos de desenvolvimento sustentável. Brasília, DF: O Ministério, 2018b. Disponível em: https://bvsms.saude.gov.br/bvs/publicacoes/saude_brasil_2017_analise situacao saude desafios objetivos desenvolvimento sustetantavel.pdf. Acesso em: 5 dez. 2020. 
BRASIL. Ministério da Saúde. Secretaria de Vigilância em Saúde. Doença de Chagas Aguda e distribuição espacial dos triatomíneos de importância epidemiológica, Brasil 2012 a 2016. Boletim Epidemiológico, Brasília, DF, v. 50, n. 2, p. 1-10, jan. 2019.

BRASIL. Ministério da Saúde. Secretaria de Vigilância em Saúde. Doença de Chagas: 14 de abril - Dia Mundial. Boletim Epidemiológico, Brasília, DF, v. 51, n. esp., p. 1-43, abr. 2020a.

BRASIL. Ministério da Saúde. Portaria $n^{\circ} 264$, de 17 de fevereiro de 2020. Altera a Portaria de Consolidação $n^{\circ}$ 4/GM/MS, de 28 de setembro de 2017, para incluir a doença de Chagas crônica, na Lista Nacional de Notificação Compulsória de doenças, agravos e eventos de saúde pública nos serviços de saúde públicos e privados em todo o território nacional. Diário Oficial da União: seção 1, Brasília, DF, n. 35, p. 97,19 fev. 2020b.

CALDAS, Cristina. Há cem anos a doença de Chagas foi descoberta. Ciência e Cultura, São Paulo, v. 60 , n. 2, p. 10-11, 2008. Disponível em: http://cienciaecultura.bvs.br/scielo.php?script=sci_arttext\&pid =S0009-67252008000200005. Acesso em: 22 set. 2021.

COORDENAÇÃO DE APERFEIÇOAMENTO DE PESSOAL DE NÍVEL SUPERIOR (CAPES). Geocapes. Brasília, DF: Capes, c2020. Disponível em: https://geocapes.capes.gov.br/geocapes/. Acesso em: 20 dez. 2020.

CARVALHO, Marília Sá; COELI, Cláudia Medina; LIMA, Luciana Dias de. Mulheres no mundo da ciência e publicação científica. Cadernos de Saúde Pública, Rio de Janeiro, v. 34, n. 3, p. 1-3, 2018. Editorial. DOI: https://doi.org/10.1590/0102-311X00025018. Disponível: http://www.scielo.br/pdf/csp/v34n3/1678-4464-csp-3403-e00025018.pdf. Acesso em: 10 abr. 2019.

CONSELHO NACIONAL DE DESENVOLVIMENTO CIENTÍFICO E TECNOLÓGICO (CNPQ). Plataforma Lattes. Brasília, DF: CNPq, c2020. Disponível em: http://lattes.cnpq.br/. Acesso em: 5 dez. 2020.

CROSS, Di; THOMSON, Simon; SIBCLAIR Alexandra. Research in Brazil: a report for CAPES by Clarivate Analytics. [S. I.]: Clarivate Analytics, 2017. Disponível em: https://www.aguia.usp.br/wp-content/ uploads/2018/01/Relat\%c3\%b3rio-Clarivate-Capes-InCites-Brasil-2018.pdf. Acesso em: 22 set. 2021.

DEPARTAMENTO DE INFORMÁTICA DO SISTEMA ÚNICO DE SAÚDE (DATASUS). Mortalidade desde 1996 pela CID-10: doença de Chagas, 2015 a 2019. Brasília, DF: DATASUS, c2020. Disponível em: https:// datasus.saude.gov.br/informacoes-de-saude-tabnet/. Acesso em: 10 dez. 2020.

DIAS, João Carlos Pinto. Globalização, iniquidade e doença de Chagas. Cadernos de Saúde Pública, Rio de Janeiro, v. 23, p. 13s-22s, 2007. Supl. 1. DOI: http://dx.doi.org/10.1590/S0102-311X2007001300003. Disponível em: https://www.scielo.br/j/csp/a/Ftv38v6jcYkbjKvPLJdHXKM/?lang=pt. Acesso em: 31 mar. 2019.

DIAS, João Carlos Pinto et al. Mudanças no paradigma da conduta clínica e terapêutica da doença de Chagas: avanços e perspectivas na busca da integralidade da saúde. Epidemiologia e Serviços de Saúde, Brasília, DF, v. 25, n. Especial, p. 87-90, 2016a. DOI: https://doi.org/10.5123/S1679-49742016000500003. Disponível em: https://www.scielo.br/j/ress/a/jBwFdrNhQjcpKJSc5StyScB/?lang=pt. Acesso em: 31 mar. 2019.

DIAS, João Carlos Pinto et al. II Consenso Brasileiro em Doença de Chagas, 2015. Epidemiologia e Serviços de Saúde, Brasília, DF. v. 25, n. Especial, p. 7-86, 2016b. DOI: http://dx.doi.org/10.5123/s167949742016000500002. Disponível em: https://www.scielo.br/j/ress/a/JrVJ3sYXSCYbvZdh8cH4Dqy/?lang=pt. Acesso em: 10 jan. 2019.

ELSEVIER. Gender in the global research landscape: analysis of research performance through a gender lens across 20 years, 12 geographies, and 27 subject areas. [S. I.]: Elsevier, 2017. (Elsevier's Research Intelligence).

FILIPPO, Daniela De; FERNÁNDEZ, María Teresa. Bibliometría: importancia de los indicadores bibliométricos. In: ALBORNOZ, M. (ed.). El estado de la ciencia: principales indicadores de ciencia y tecnología Iberoamericanos/interamericanos. Buenos Aires: Editora Artes Gráfica Integradas, 2002. p. 69-76.

GONZÁLEZ-ALCAIDE, Gregorio et al. Scientific authorships and collaboration network analysis on Chagas disease: papers indexed in PubMed (1940-2009). Revista do Instituto de Medicina Tropical de São Paulo, São Paulo, v. 54, n. 4, p. 219-228, 2012. DOI: https://doi.org/10.1590/S0036-46652012000400007. Disponível em: https://www.scielo.br/j/rimtsp/a/3wXRcK8j4Pfh4DMjct69Jpc/?lang=en. Acesso em: 22 set. 2021. 
HAYASHI, Maria Cristina Piumbato et al. Um estudo bibliométrico da produção científica sobre a educação jesuítica no Brasil colonial. Biblios, Tacna, v. 8, n. 27, p. 1-18, mar. 2007. Disponível em: https://dialnet. unirioja.es/servlet/articulo?codigo=2281795. Acesso em: 22 set. 2021.

KROPF, Simone Petraglia; AZEVEDO, Nara; FERREIRA, Luiz Otávio. Doença de Chagas: a construção de um fato científico e de um problema de saúde pública no Brasil. Ciência \& Saúde Coletiva, Rio de Janeiro, v. 5, n. 2, p. 347-365, 2000. DOI: https://doi.org/10.1590/S1413-81232000000200009. Disponivel em: https://www. scielo.br/j/csc/a/79tmmamRSjn9zVQYkzM6ycQ/?lang=pt. Acesso em: 22 set. 2021.

LEE, Hikyoung; LEE, Kathy. Publish (in international indexed journals) or perish: neoliberal ideology in a Korean university. Language Policy, Berlin, v. 12, n. 3, p. 215-230, apr. 2013. DOI: https://doi.org/10.1007/ s10993-012-9267-2. Disponível em: https://link.springer.com/article/10.1007\%2Fs10993-012-9267-2. Acesso em: 10 jan. 2019.

MALAFAIA, Guilherme. Análise de informações sobre a doença de Chagas disponíveis em websites brasileiros na rede mundial de computadores (internet). Arquivos Brasileiros de Ciências da Saúde, Santo André, v. 34, n. 3, p. 188-195, 2009. DOI: https://doi.org/10.7322/abcs.v34i3.123. Disponível em: https://www. portalnepas.org.br/abcs/article/view/123. Acesso em: 22 set. 2021.

MENEGHINI, Rogerio; PACKER, Abel L. Is there science beyond English? Initiatives to increase the quality and visibility of non-English publications might help to break down language barriers in scientific communication. EMBO Reports, Londres, v. 8, n. 2, p. 112-116, 2007. DOI: https://dx.doi.org/10.1038\%2Fsj. embor.7400906. Disponível em: https://www.ncbi.nlm.nih.gov/pmc/articles/PMC1796769/pdf/7400906.pdf. Acesso em: 22 fev. 2019.

MIOTTO, Mariana Monteiro Barros; BARCELLOS, Ludmilla Awad. Contribuição das Ciências Sociais nas práticas de Saúde Pública. Revista Brasileira de Pesquisa em Saúde, Vitória, v. 11, n. 2, p. 43-48, 2009. Disponível em: https://www.periodicos.ufes.br/rbps/article/download/426/301/355. Acesso em: 22 set. 2021.

OLIVEIRA, Roberta Gondim de. Sentidos das doenças negligenciadas na agenda da saúde global: o lugar de populações e territórios. Ciência \& Saúde Coletiva, Rio de Janeiro, v. 23, n. 7, p. 2291-2302, 2018. DOI: https://doi.org/10.1590/1413-81232018237.09042018. Disponível em: https://www.scielo.br/j/csc/a/ Zs9vNx7xqcc3XrjsmdSCRFm/abstract/?lang=pt. Acesso em: 22 set. 2021.

OLIVEIRA, Talita Rodrigues; LEITE, Fernando César Lima. Produção do conhecimento sobre doenças negligenciadas: características da produção científica sobre doença de chagas na Web of Science. In: REUNIÃO ANUAL DA SOCIEDADE BRASILEIRA PARA O PROGRESSO DA CIÊNCIA, 68., 2016, São Paulo. Resumos [...], Porto Seguro: Universidade Federal do Sul da Bahia, 2016. Disponível em: http://www.sbpcnet.org.br/ livro/68ra/resumos/resumos/3225 120b340096e89b8554fc9d2e9aa328468.pdf. Acesso em: 10 mar. 2019.

ORGANIZAÇÃO DAS NAÇÕES UNIDAS (ONU). Transformando nosso mundo: Agenda 2030 para o Desenvolvimento Sustentável. Nova York: A Organização, 2015. Disponível em: https://nacoesunidas.org/ pos2015/agenda2030/. Acesso em: 20 jul. 2020.

ORGANIZAÇÃO PAN-AMERICANA DA SAÚDE (OPAS). Agenda de Saúde Sustentável para as Américas 2018-2030. Washington, DC: A Organização, set. 2017. Disponível em: http://iris.paho.org/xmlui/bitstream/ handle/123456789/49172/CSP296-por.pdf?sequence=1\&isAllowed=y. Acesso em: 20 jul. 2020.

PELLEGRINI, Alberto; GOLDBAUM, Moisés; SILVI, John. Producción de artículos científicos sobre salud en seis países de América Latina, 1973 a 1992. Revista Panamericana de Salud Pública, Washington, DC, v.1, n.1, 1997. Disponível em: https://www.scielosp.org/pdf/rpsp/1997.v1n1/23-34/es. Acesso em: 17 mar. 2019.

SANGENIS, Luis Henrique Conde et al. Transmissão da doença de Chagas por consumo de carne de caça: revisão sistemática. Revista Brasileira de Epidemiologia, São Paulo, v. 19, n. 4, p. 803-811, 2016. DOI: https://doi.org/10.1590/1980-5497201600040010. Disponível em: https://www.scielo.br/j/rbepid/al C5Z4GNTtYxxtWfRkwRrvqRM/abstract/?lang=pt. Acesso em: 22 set. 2021.

SILVA, Ayres Fran Silva; LUZ JUNIOR, Geraldo Eduardo. Prospecção tecnológica: potencialidades de diagnósticos para doenças de chagas. Revista Brasileira de Inovação Tecnológica em Saúde, Natal, v. 6, n. 2, p. 15-31, 2016. DOI: https://doi.org/10.18816/r-bits.v6i2.7400. Disponível em: https://periodicos.ufrn.br/reb/ article/view/7400. Acesso em: 22 set. 2021.

SILVEIRA, Antonio Carlos et al. O controle da doença de Chagas nos países do Cone Sul da América: história de uma iniciativa internacional, 1991-2001. [Uberaba]: Opas, 2002. 
SOBRAL, Natanael Vitor et al. O Qualis e os periódicos científicos na produção de conhecimento em doenças tropicais negligenciadas. Informação \& Sociedade: Estudos, João Pessoa, v. 30, n. 3, p. 1-24, 2020. DOI: https://doi.org/10.22478/ufpb.1809-4783.2020v30n3.52054. Disponível em: https://periodicos.ufpb.br/index. php/ies/article/view/52054. Acesso em: 22 set. 2021.

SOBRAL, Natanael Vitor et al. Produção científica sobre medicina tropical na América Latina: um olhar sobre a web of science. In: ENCONTRO BRASILEIRO DE BIBLIOMETRIA E CIENTOMETRIA, 5., 2016, São Paulo. Anais [...]. São Paulo: Universidade de São Paulo, 2016.

SOBRAL, Natanael Vitor.; SILVA, Fábio Mascarenhas; MIRANDA, Zeny Duarte de. Perfil da produção científica em Medicina Tropical na América Latina: análise do termo "Tropical Medicine" na Web of Science. Em Questão, Porto Alegre, v. 23, n. Especial, p. 31-49, 2017. DOI: http://dx.doi.org/10.19132/1808-5245230.31-49. Disponível em: https://seer.ufrgs.br/index.php/EmQuestao/article/view/68093. Acesso em: 30 de jan. 2020.

VIEIRA, Ricardo Quintão; SANNA, Maria Cristina. O uso do estudo bibliométrico pelos pesquisadores da saúde em periódicos científicos digitais brasileiros. In: CONGRESSO BRASILEIRO DE BIBLIOTECONOMIA, DOCUMENTAÇÃO E CIÊNCIA DA INFORMAÇÃO, 15., 2013, Santa Catarina. Anais [...] São Paulo: Federação Brasileira de Associações de Bibliotecários, Cientistas da Informação e Instituições, 2013.

VILLELA, Marcos Marreiro et al. Avaliação do Programa de Controle da Doença de Chagas em relação à presença de Panstrongylus megistus na região centro-oeste do Estado de Minas Gerais, Brasil. Cadernos de Saúde Pública, Rio de Janeiro, v. 25, n.4, abr. 2009. DOI: https://doi.org/10.1590/S0102-311X2009000400022. Disponível em: http://www.scielo.br/pdf/csp/v25n4/22.pdf. Acesso em: 10 jun. 2019.

WESTPHALEN, Elizabeth Visone Nunes et al. Aspectos epidemiológicos e históricos do controle da doença de Chagas no Continente Americano. BEPA. Boletim Epidemiológico Paulista, São Paulo, v. 9, n. 105, p. 1835, 2012. 